\title{
Automated processing of whole blood samples for the determination of immunosuppressants by liquid chromatography tandem-mass spectrometry
}

\author{
Michael Vogeser ${ }^{1, *}$ and Ute Spöhrer ${ }^{2}$ \\ ${ }^{1}$ Institute of Clinical Chemistry, \\ ${ }^{2}$ Hospital Pharmacy, \\ Hospital of the University of Munich, \\ Munich, Germany
}

\begin{abstract}
Background: Liquid chromatography tandem-mass spectrometry (LC-MS/MS) is an efficient technology for routine determination of immunosuppressants in whole blood; however, time-consuming manual sample preparation remains a significant limitation of this technique.
\end{abstract}

Methods: Using a commercially available robotic pipetting system (Tecan Freedom EVO), we developed an automated sample-preparation protocol for quantification of tacrolimus in whole blood by LC-MS/ MS. Barcode reading, sample resuspension, transfer of whole blood aliquots into a deep-well plate, addition of internal standard solution, mixing, and protein precipitation by addition of an organic solvent is performed by the robotic system. After centrifugation of the plate, the deproteinized supernatants are submitted to on-line solid phase extraction, using column switching prior to LC-MS/MS analysis. The only manual actions within the entire process are decapping of the tubes, and transfer of the deep-well plate from the robotic system to a centrifuge and finally to the HPLC autosampler. Whole blood pools were used to assess the reproducibility of the entire analytical system for measuring tacrolimus concentrations.

Results: A total coefficient of variation of $1.7 \%$ was found for the entire automated analytical process $(\mathrm{n}=40$; mean tacrolimus concentration, $5.3 \mu \mathrm{g} / \mathrm{L}$ ). Close agreement between tacrolimus results obtained after manual and automated sample preparation was observed.

Conclusions: The analytical system described here, comprising automated protein precipitation, on-line solid phase extraction and LC-MS/MS analysis, is convenient and precise, and minimizes hands-on time and the risk of mistakes in the quantification of whole blood immunosuppressant concentrations compared to conventional methods.

Clin Chem Lab Med 2006;44:1126-30.

*Corresponding author: Michael Vogeser, MD, Institute of Clinical Chemistry, Hospital of the University of Munich, Marchioninistr. 15, 81377 Munich, Germany Phone: + 49-89-70953221, Fax: + 49-89-70953240, E-mail: michael.vogeser@med.uni-muenchen.de
Keywords: automation; immunosuppressants; liquid chromatography tandem-mass spectrometry (LC-MS/ MS); sample preparation.

\section{Introduction}

Pharmacological suppression of immune functions represents a cornerstone in transplantation medicine. Dosage individualization according to whole-blood drug concentrations is recognized to be mandatory for the main immunosuppressants cyclosporin $A$, tacrolimus, sirolimus, and everolimus. Immunoassays still represent the predominant analytical technology used for therapeutic drug monitoring of these drugs. Reagent costs of these tests are substantial, and limitations of the analytical quality of these immunoassays in terms of specificity and reproducibility are recognized. During recent years, liquid-chromatography tandem-mass spectrometry (LC-MS/MS) has been introduced in many laboratories as an alternative technology for immunosuppressant monitoring (1). The application of this powerful technology in the clinical laboratory, however, is still limited with respect to several issues: high instrument costs; the need for development of instrument-specific analytical protocols; demanding instrument handling compared to immunoassay systems; the need for skilled technicians; and limited support by the instrument manufacturers. Compared to gas chromatographymass spectrometry, the requirements for sample clean-up are limited for LC-MS/MS. However, protein removal is mandatory and in our experience solidphase or solvent extraction is necessary for rugged and highly precise quantitative methods. While solidphase extraction can easily be automated by column switching and the application of permanently used extraction columns (2-6), manual sample handling for protein precipitation leads to a substantial workload for large-scale immunosuppressant monitoring by LC-MS/MS. Automation of this first step in sample work-up represents a particular challenge with whole blood as the sample material because sedimentation of blood cells is observed within a few minutes, but complete resuspension must be achieved immediately before quantitative sample pipetting.

Here, we describe the development, optimization and validation of a comprehensive automated analytical system for immunosuppressant monitoring based on LC-MS/MS. 


\section{Methods}

\section{Instruments}

Pipetting system A Tecan Freedom EVO (Tecan, Männedorf, Switzerland) pipetting system was used. This system allows flexible positioning of elements on a worktable of $70 \mathrm{~cm} \times 90 \mathrm{~cm}$. In the configuration used, one robotic arm (liquid handling arm) controls four independent carriers for disposable pipetting tips in the $x-, y-$, and $z$-dimensions. Furthermore, the system is equipped with a washing station, a chilled reagent carrier for up to six troughs, a plate carrier on a horizontal shaker, an additional static plate carrier, a barcode reader, and six sample trays for blood collection tubes (Figure 1). The system is controlled by Evoware software, which allows detailed programming of all movements of the liquid handling arm and of pipetting actions. The system is equipped with a liquid-level detection system and a clot detection system.

Disposables used were 96-position 2-mL deep-well plates (Corning, Corning, NY, USA; catalogue no. 3958), adhesive sealing tape (Corning; catalogue no. 6569), and 1000- $\mu$ L conductive pipetting tips (Tecan).

Centrifuge A Hettich Rotanta 460 (Hettich, Tuttlingen, Germany) benchtop centrifuge was used for protein precipitation.

HPLC and MS/MS system A Waters Alliance 2795 HPLC separation module (Waters, Milford, MA, USA) consisting of an autosampler, a gradient pump and a column oven was used for sample injection and clean-up. For analytical chromatography, an additional HPLC pump (Waters 600E) was used, coupled via a T-piece with a split of approximately 1:10 to a Micromass Quattro Ultima Pt MS/MS system (Waters).

On-line solid-phase extraction protocol, analytical chromatography and mass spectrometric conditions The on-line solid phase extraction protocol used has been described previously (4). In brief, a Waters Oasis $\mathrm{HLB}^{\circledR}$ sample preparation column $(2.1 \times 20 \mathrm{~mm}$, particle size $25 \mu \mathrm{m})$ is used with a sixport high-pressure switching valve (Rheodyne, Rohnert Park, CA, USA), which is installed into the Waters 2795 separation module and controlled by Micromass MassLynx 4.0 software (Waters). The operating procedure for the clean-up system consists of three steps. First, $15 \mu \mathrm{L}$ of deproteinized sample is injected into the system by the autosampler and transferred onto the Oasis HLB ${ }^{\circledR}$ extraction column. Analytes are adsorbed onto the column, whereas potentially interfering hydrophilic matrix compounds are washed directly into the waste by mobile phase consisting of water/methanol (95:5, $\mathrm{v} / \mathrm{v}$ ) delivered at a flow rate of $4 \mathrm{~mL} / \mathrm{min}$ for $1 \mathrm{~min}$. In parallel, the analytical column (Reprosil pur C18-AQ, $125 \times 2 \mathrm{~mm}$, $5 \mu \mathrm{m}$; Maisch, Ammerbuch, Germany) is equilibrated with methanol/0.5 mM ammonium acetate $(90: 10, \mathrm{v} / \mathrm{v})$ at a flow rate of $0.8 \mathrm{~mL} / \mathrm{min}$. Subsequently, the six-port valve is switched to its initial position and the extract is eluted in back-flush mode from the Oasis $\mathrm{HLB}^{\circledR}$ column and transferred onto the analytical separation column using the equilibration mobile phase at $0.8 \mathrm{~mL} / \mathrm{min}$. The valve is switched back to its initial position and isocratic HPLC separation is performed. Simultaneously, the extraction column is washed with pure methanol at $4 \mathrm{~mL} / \mathrm{min}$ for $1 \mathrm{~min}$ and subsequently re-equilibrated with water/methanol $(95: 5, v / v)$. Both the extraction and the analytical columns are kept at $38^{\circ} \mathrm{C}$ in a column oven. The configuration of the chromatographic system is shown in Figure 2.

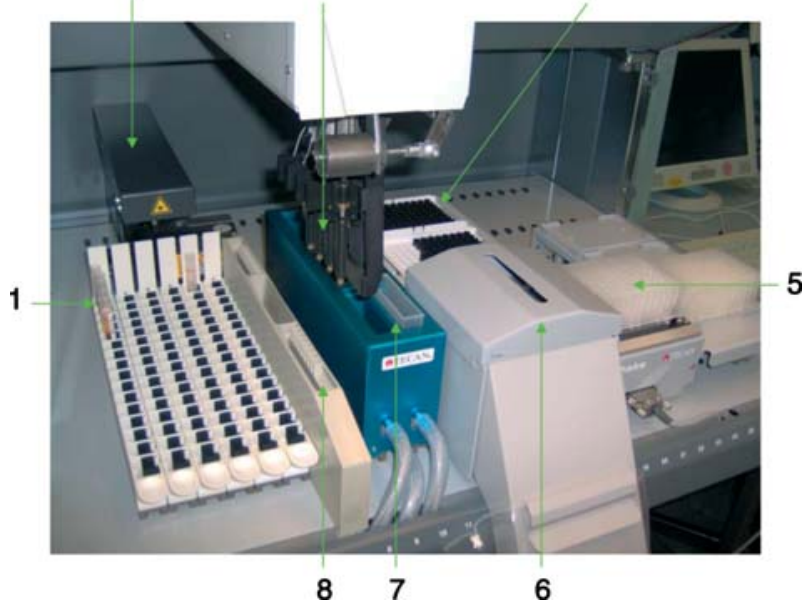

Figure 1 Worktable of the Tecan Freedom EVO pipetting system. 1, sample trays with decapped whole-blood samples; 2, barcode reader; 3, liquid handling arm with four pipette carriers; 4, disposable pipetting tips; 5, 96-position 2-mL deep-well plate on a horizontal shaker; 6 , dropping station for used pipette tips; 7 , chilled reagent troughs (internal standard and precipitation solution, respectively); and 8, washing station.

Electrospray atmospheric pressure ionization in the positive mode is used for analyte detection; the source parameters are tuned to obtain the ammonium adduct ions of tacrolimus and the internal standard ascomycin, respectively (821 and $809 \mathrm{~m} / \mathrm{z}$ ). The following settings result in optimum ion yield: capillary voltage, $3.0 \mathrm{kV}$; cone voltage, $25 \mathrm{~V}$; source temperature $80^{\circ} \mathrm{C}$; desolvation temperature $230^{\circ} \mathrm{C}$; nitrogen flow, approximately $630 \mathrm{~mL} / \mathrm{min}$; and nebulizer gas flow, approximately $75 \mathrm{~mL} / \mathrm{min}$. The collision energy with argon as the collision gas is $20 \mathrm{eV}$. The following ion transitions are used for multiple reaction monitoring (MRM): tacrolimus $821 \rightarrow 768 \mathrm{~m} / \mathrm{z}$, and ascomycin (internal standard) $809 \rightarrow$ $756 \mathrm{~m} / \mathrm{z}$. The retention times of tacrolimus and the internal standard are approximately $2.3 \mathrm{~min}$, and a complete analysis cycle including solid phase extraction (SPE) and separation takes $4.5 \mathrm{~min}$. A representative chromatogram for a whole blood sample is shown in Figure 3 (tacrolimus concentration $5.3 \mu \mathrm{g} / \mathrm{L})$. Linear six-point calibration is routinely used $(2.1-38.8 \mu \mathrm{g} / \mathrm{L})$ and the lower limit of quantification is
A

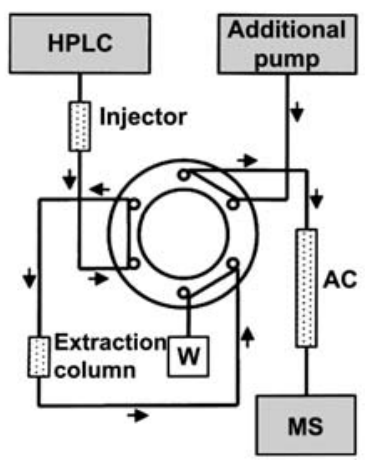

B

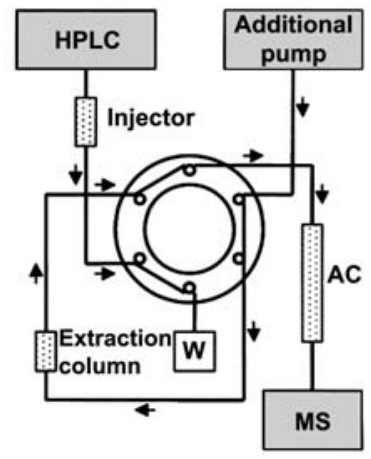

Figure 2 Configuration of the on-line solid-phase extraction and analytical chromatography devices (W, waste; AC, analytical column). 


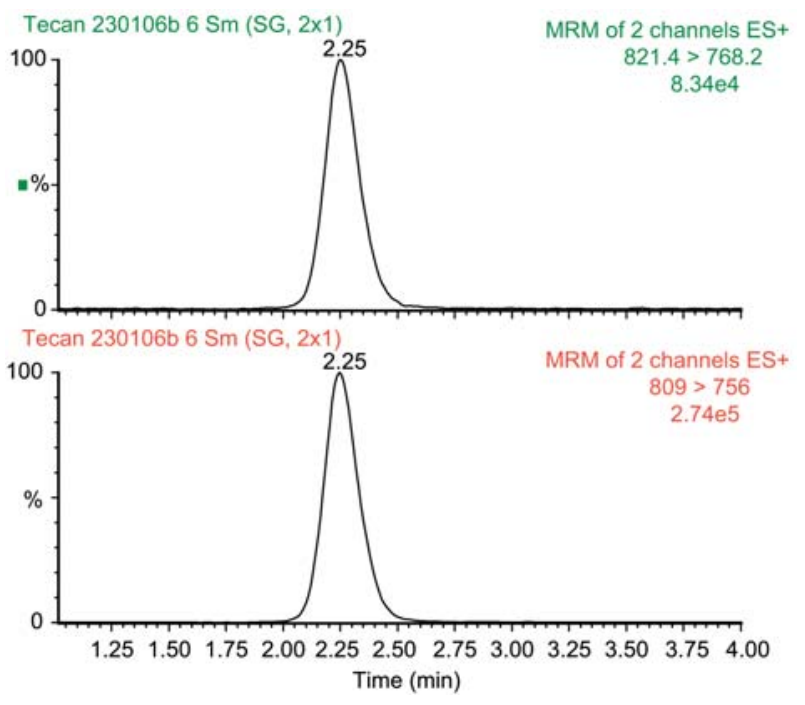

Figure 3 Determination of whole-blood tacrolimus concentrations using the described LC-MS/MS method using automated pipetting and on-line solid-phase extraction. Representative chromatogram (tacrolimus concentration $5.3 \mu \mathrm{g} / \mathrm{L})$. Upper trace, tacrolimus; lower trace ascomycin (internal standard).

$1.0 \mu \mathrm{g} / \mathrm{L}$. No relevant ion suppression has been observed (comparison of internal standard peak areas in patient samples and in pure solutions). This on-line solid-phase extraction/LC-MS/MS configuration was used for tacrolimus measurement with either manual or automated sample preparation.

\section{Method development}

To establish a precision benchmark for automation, we assessed the reproducibility of the standard semi-automated sample preparation protocol with manual protein precipitation before we started development of the fully automated method. For this purpose, whole blood pools from patients treated with tacrolimus were analyzed in determination of ten replicates. The protocol for manual sample preparation was as follows. Polypropylene reaction tubes $(2 \mathrm{~mL})$ were labeled with the identification number for the whole blood samples. Using a "multipette", $150 \mu \mathrm{L}$ of precipitation solution [methanol/zinc sulfate solution $(8.9 \mathrm{~g} / 100 \mathrm{~mL}$ $\mathrm{ZnSO}_{4} \cdot 7 \mathrm{H}_{2} \mathrm{O}$ ), 4:1] containing the internal standard (ascomycin, $20 \mu \mathrm{g} / \mathrm{L}$ ) was dispensed into the reaction tubes. Each sample was gently mixed overhead five times, briefly vortexed and decapped. Then $150 \mu \mathrm{L}$ of the resuspended sample was transferred into the reaction tube, which was vortexed for approximately $10 \mathrm{~s}$. After pipetting of the whole series, all reaction tubes were placed for $5 \mathrm{~min}$ on a horizontal shaker and centrifuged for $10 \mathrm{~min}(16,000 \times \mathrm{g})$ in a benchtop centrifuge. Meanwhile, 2-mL HPLC vials were labeled and adapted with low-volume inserts. Then $200 \mu \mathrm{L}$ of sample supernatant was transferred from the reaction tube into the HPLC vial. The vials were capped and placed into the HPLC autosampler tray. An MS/MS sample list was created by writing the sample identification for the HPLC vials into a MassLynx series file. Total expenditure for consumables was approximately $€ 2.50 /$ sample (manufacturer's list prices). The turnaround time for preparation of 20 samples was $45 \mathrm{~min}$ (start of the process until start of the analytical LC-MS/MS run). Using this manual precipitation protocol, intra-assay coefficients of variation (CVs) for tacrolimus between $2.4 \%$ and $5.3 \%$ were observed in five series (pool concentrations between 4.6 and $9.9 \mu \mathrm{g} / \mathrm{L} ; \mathrm{n}=10$ replicates). Consequently, the precision goal for automation was defined as an intra-assay CV of less than $3.0 \%$.

After these benchmark investigations of the manual sample preparation, the corresponding steps of the protocol were programmed to the pipetting system; the practicality and reproducibility of the automated sample preparation were initially investigated. CVs of approximately $15 \%$ were observed. Subsequently, numerous settings of the pipetting steps were optimized, including the draw speeds, the dispensing and aspiration delays, and the tip positions for aspiration and dispensing in the sample tubes and deep wells (during resuspension, aliquot transfer, and dispensing of internal standard and protein precipitation solution). In particular, it was necessary to dispense the internal standard dissolved in a non-precipitating solution rather than together with the precipitation solution, as in the manual sample preparation protocol. Using the horizontal shaker of the Tecan instrument, two-step addition of the internal standard and precipitation solution yields a homogenous distribution of the internal standard within the whole blood samples. The optimized final version was validated. The steps of this final pipetting protocol are given in Table 1. The start-up procedures for the pipetting system involve: placing two 96-position pipette tip trays onto the instrument; labeling and placing two 96-position 2-mL deep-well plates onto the instrument (one onto the shaker station for sample preparation, one on a fixed tray holder); filling internal standard solution into a chilled reagent trough [ascomycin $20 \mu \mathrm{g} / \mathrm{L}$ in methanol/water (20:80)]; filling precipitation solution into a chilled reagent trough [methanol/zinc sulfate solution $\left.\left(8.9 \mathrm{~g} / 100 \mathrm{~mL} \mathrm{ZnSO}_{4} \cdot 7 \mathrm{H}_{2} \mathrm{O}\right), 4: 1\right]$; and checking the level of system fluid (distilled water) and liquid waste.

After start-up, the turnaround time for preparation of 20 samples (from start of decapping until start of the LC-MS/ MS run) was approximately $30 \mathrm{~min}$ (18 min, pipetting; $10 \mathrm{~min}$, centrifugation; $1 \mathrm{~min}$, transfer time). Total expenditure for consumables was approximately $€ 0.40 /$ sample.

\section{System validation}

Imprecision studies Residual EDTA-anticoagulated whole blood samples from patients treated with tacrolimus were used to prepare pools in $50-\mathrm{mL}$ polypropylene tubes. After mixing and equilibration for $30 \mathrm{~min}$, these pools were aliquoted $(2 \mathrm{~mL})$ into blood sampling tubes routinely used for immunosuppressant monitoring (EDTA Monovette; Sarstedt, Nümbrecht, Germany). The use of residual patient sample material for this study was approved by the institutional Review Board. Analyses were performed within $48 \mathrm{~h}$ after preparation of the pools, which were kept at $4^{\circ} \mathrm{C}$.

For calibration of the tacrolimus assay, commercially available materials were used (Chromsystems, Munich, Germany; six-point calibrator set). These whole blood-based materials were transferred to blood sampling tubes and were processed identically to pool and patient samples.

To investigate the intra-assay imprecision of the fully automated analytical system, ten aliquots of one pool were analyzed in four series on two subsequent days. In the different series, all capped samples were first resuspended by manual overhead rotation of the sample trays. Then the samples were allowed to sediment for $0.5,1,1.5$, and $2 \mathrm{~h}$, respectively, in the different series. Before starting the series, five of the pool samples were manually resuspended before starting the automated sample preparation procedure. In this way, five of ten samples had sedimented for $30,60,90$, and $120 \mathrm{~min}$, respectively, in the different series before starting 
sample preparation. A total CV was calculated for all 40 determinations.

Method comparison Whole blood samples $(n=20)$ sent for routine quantification of tacrolimus were used for a method comparison study. Measurement was first performed using the automated procedure followed by on-line solid phase

Table 1 Protocol for the automated pipetting system for sample preparation for LC-MS/MS analysis of tacrolimus in whole blood.

\section{User actions}

1. Barcode labeled whole-blood patient samples are placed into 16-position sample trays. Samples are resuspended by gently rotating the trays overhead. Tubes are decapped manually and the trays are positioned into the pipetting system.

2. The safety door is closed and the preparation process is started by entering the number of samples loaded into the system software.

Actions of the pipetting system

3. Sample barcodes are read tray by tray (the trays are moved along a barcode-reader by the system).

4. Internal standard solution $(20 \mu \mathrm{g} / \mathrm{L}$ ascomycin in methanol/water 20:80, $100 \mu \mathrm{L}$ ) is pipetted from the reagent trough into the wells of a 96-position 2-mL deep-well plate using separate tips for each well.

5. Four whole blood samples are resuspended simultaneously using disposable tips. First $600 \mu \mathrm{L}$ from each sample is aspirated at the bottom position of the sample tube and dispensed on the upper liquid level with level tracking. Subsequently, $600 \mu \mathrm{L}$ of the sample is aspirated at the upper liquid level and dispensed at the bottom position. These two mixing steps are replicated. Aspiration is carried out slowly $(200 \mu \mathrm{L} / \mathrm{s})$ and dispensing rapidly $(1200 \mu \mathrm{L} / \mathrm{s})$.

6. After changing the tips, $150 \mu \mathrm{L}$ of the samples are pipetted from $50 \%$ of the upper liquid level into the wells of the deep-well plate in a sequence corresponding to the sample sequence in the trays. After completion of pipetting of the whole series, the plate is shaken (1200 rev/min, $1 \mathrm{~min}$ ).

7. A sample data file is written in Microsoft Excel format giving the deep-well position and the corresponding barcode identification for each sample.

8. Using new tips for each well, $300 \mu \mathrm{L}$ of the precipitation solution [methanol/zinc sulfate solution $(8.9 \mathrm{~g} /$ $100 \mathrm{~mL} \mathrm{ZnSO}_{4} \cdot 7 \mathrm{H}_{2} \mathrm{O}$ ), 4:1] is pipetted into the sample wells in three steps, with $100 \mu \mathrm{L}$ dispensed into the bottom of the well, at $50 \%$ of the liquid level, and onto the sample surface, respectively.

9. The deep-well plate is shaken for $2 \mathrm{~min}(1200 \mathrm{rev} / \mathrm{min}$ ).

10. Into a second deep-well plate positioned on the instrument, $450 \mu \mathrm{L}$ of system water is dispensed into a number of wells corresponding to the number of samples processed ("tare plate" for balancing in the subsequent centrifugation step).

User actions

11. The sample plate is sealed with adhesive film, the sample plate and tare plate are placed manually into the centrifuge and centrifugation for $10 \mathrm{~min}$ at $6500 \times \mathrm{g}$ is initiated.

12. The Excel sample list is transferred from the PC of the pipetting system into a template sample list of the MS/MS system using a USB memory stick.

13. After centrifugation, the deep-well plate is placed in the autosampler of the LC system. The analytical LCMS/MS series with on-line solid-phase extraction is started. extraction and LC-MS/MS analysis. Using the identical calibration materials, a second analytical series was run using the manual protein precipitation protocol. Tacrolimus results obtained from the two analytical runs were compared according to Passing and Bablok and by calculation of the respective mean concentrations. To test for significant differences between the preparation methods the unpaired t-test was used.

To compare analyte recovery for the manual and automated precipitation protocols, the mean peak areas for tacrolimus from multiple reaction monitoring traces for identical pool samples processed with the different protocols were calculated

\section{Results}

Using the automated protocol, a total tacrolimus CV of $1.7 \%$ was found for the entire analytical method (concentration $5.3 \mu \mathrm{g} / \mathrm{L} ; 40$ determinations in four independent analytical runs performed on two subsequent days). The intra-assay $\mathrm{CVs}(\mathrm{n}=10)$ ranged from 1.0 to $1.3 \%$.

In the method comparison study, the mean tacrolimus concentration found for 20 samples was 7.5 $\mu \mathrm{g} / \mathrm{L}$ for manual protein precipitation and $7.2 \mu \mathrm{g} / \mathrm{L}$ for the automated protocol (concentration range 0-14.9 $\mu \mathrm{g} / \mathrm{L})$; the difference was not statistically significant. Data analysis according to Passing and Bablok revealed the following equation:

\section{[manual protocol $]=1.05 \times[$ automated protocol $]$}

$$
-0.1[\mu \mathrm{g} / \mathrm{L}] \text {, }
$$

with a Pearson coefficient of $r=0.989$.

Comparison of the analyte recovery based on mean tacrolimus peak areas found in pool samples did not show significant differences between the manual and automated precipitation protocols.

During method development and validation, the entire analytical system proved rugged and convenient, and no technical malfunctions occurred.

\section{Discussion}

We have developed a method that allows for the first time direct automated processing of large series of whole blood samples for immunosuppressant monitoring by LC-MS/MS; the entire analytical system proved to be highly precise and convenient. The systems comprises sample dispensing and protein precipitation steps using a pipetting robot, automated solid-phase extraction of deproteinized samples by on-line solid-phase extraction with column-switching, and LC-MS/MS analyte detection.

Automation of quantitative analyses for whole blood samples is a particular challenge because sedimentation of blood cells occurs within minutes, particularly in patients in the acute phase response. We were able to achieve complete automated resuspension of the samples before quantitative pipetting by repeated aspiration and dispensing of the sample material using disposable 1-mL pipetting tips. Careful 
optimization of the liquid handling steps finally resulted in a highly precise analytical solution, which outperformed manual sample preparation.

Full automation of immunosuppressant quantification by LC-MS/MS with a pipetting robot requires substantial capital expenditure; the list price of the instrument described here is approximately $€ 70,000$. However, automation offers important improvements in terms of both routine laboratory workflow and anaIytical quality. Besides improved reproducibility, the risk of labeling errors is avoided by our automation protocol. Therefore, the reliability of the entire analytical process is substantially improved compared to manual handling. Importantly, automation minimizes the hands-on time of specialized technicians, as well as direct handling of infectious material by operators. Running costs are lower for automated compared to manual handling, because single HPLC vials are substantially more expensive compared to a 96-position deep-well plate; approximately $€ 2 /$ sample can be saved.

The rate-limiting step of the entire analytical process described here is the chromatographic separation of the samples, which is required for highly sensitive LC-MS/MS analyses. Minimizing the chromatographic separation usually leads to ion-suppression effects and limited reliability and ruggedness of the method. We have had very favorable experiences with the on-line solid-phase extraction principle that is integrated into the comprehensive method described here. The LC-instrument time required for this clean-up is $60 \mathrm{~s} /$ sample; however, contamination of ion source parts is minimized with this protocol. With daily series of approximately 70 samples, downtime for cleaning is less than $\sim 3 \mathrm{~h} /$ year, with 1 day for scheduled maintenance of the entire LC-MS/MS system. The lifetime of the extraction columns is approximately 1000 injections. Off-line solid-phase extraction or even solvent extraction as an alternative approach for sample clean-up would require severalfold longer hands-on time. Mere protein precipitation, on the other hand, is not sufficient to maintain good performance of the LC-MS/MS systems for the analysis of large routine series.

Validation of our innovative analytical system was performed with tacrolimus as the target analyte, but it can reasonably be assumed that similar data would be obtained for the simultaneous analysis of sirolimus, everolimus and cyclosporin $A$ as well.

In summary, the comprehensive automated system for measurement of tacrolimus based on LC-MS/MS described in this report offers substantial advantages compared to the semi-automated methods used so far, and can be recommended for routine use in the clinical laboratory.

\section{References}

1. Taylor PJ. Therapeutic drug monitoring of immunosuppressant drugs by high-performance liquid chromatography-mass spectrometry. Ther Drug Monit 2004;26:215-9.

2. Lensmeyer GL, Poquette MA. Therapeutic monitoring of tacrolimus concentrations in blood: semi-automated extraction and liquid chromatography-electrospray ionization mass spectrometry. Ther Drug Monit 2001;23: 239-49.

3. Streit F, Armstrong VW, Oellerich M. Rapid liquid chromatography-tandem mass spectrometry routine method for simultaneous determination of sirolimus, everolimus, tacrolimus, and cyclosporin A in whole blood. Clin Chem 2002;48:955-8.

4. Vogeser M, Fleischer C, Meiser B, Groetzner J, Spöhrer $U$, Seidel D. Quantification of sirolimus by liquid chromatography-tandem mass spectrometry using on-line solid-phase extraction. Clin Chem Lab Med 2002;40:40-5.

5. Ceglarek U, Lembcke J, Fiedler GM, Werner M, Witzigmann $\mathrm{H}$, Hauss JP, et al. Rapid simultaneous quantification of immunosuppressants in transplant patients by turbulent flow chromatography combined with tandem mass spectrometry. Clin Chim Acta 2004;346:181-90.

6. Koal T, Deters M, Casetta B, Kaever V. Simultaneous determination of four immunosuppressants by means of high speed and robust on-line solid phase extraction-high performance liquid chromatography-tandem mass spectrometry. J Chromatogr B Analyt Technol Biomed Life Sci 2004;805:215-22.

Received May 3, 2006, accepted June 13, 2006 